\title{
Sin campo para la vieja banca: indicadores de aceptación para la banca en línea en China
}

\author{
No Country for Old Banking: \\ Acceptance Index for Online Banking in China
}

Sem espaço para bancos velhos:

indicadores de aceitação para o internet banking da China

\author{
Po Chun Lee Yeh \\ Instituto de Altos Estudios Nacionales. Quito, Ecuador \\ pochunlee@gmail.com \\ https://orcid.org/0000-003-3216-7596

\section{Yong-ji Guo} \\ Asset Management Association of Jiangsu. Nanjing, China \\ guoyongji1221@126.com \\ https://orcid.org/0000-0002-5015-2710
}

DOI: https://doi.org/10.32719/25506641.2021.9.2

Recibido: 27 de junio 2020 • Revisado: 27 de julio 2020

Aceptado: 10 de agosto de 2020

Artículo de investigación

Licencia Creative Commons

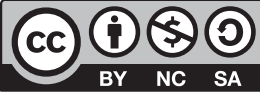




\section{Resumen}

La innovación tecnológica y los cambios en los estilos de vida (particularmente debido a la pandemia de la COVID-19) han hecho que las interacciones entre los consumidores y los bancos cambien súbitamente. Este documento estudia múltiples indicadores que afectan la aceptación de la banca por internet en China, incluida la preferencia del cliente, los factores de la banca en línea, los factores sociales y otros. Se realizaron cuestionarios en línea a pequeños y microempresarios, ejecutivos de bancos comerciales y clientes bancarios. Se hizo un modelo de red neuronal artificial de datos que comprenden más de un millar de grupos. Los resultados muestran que la actitud hacia la seguridad de la cuenta, la comprensión de las ventajas de la banca por internet, la evaluación del riesgo del prestatario y la actitud del cliente hacia el sistema de información crediticia son factores relativamente importantes que afectan la aceptación de la banca por internet. La dirección de impacto de cada indicador muestra que es más probable que los clientes acepten la banca por internet si tuvieran una mejor seguridad, más conveniencia, un menor riesgo para los clientes y un sistema de información y vigilancia de crédito más desarrollado. En contraste, los indicadores para aplicaciones de teléfonos móviles, funciones auxiliares, regulaciones y operaciones de la banca en línea son relativamente menos importantes.

Palabras clave: banca por internet, red neuronal artificial, China, preferencia clientes, innovación tecnológica.

JEL: G41 Rol y efectos de los factores psicológicos, emocionales, sociales y cognitivos en la toma de decisiones en los mercados financieros.

\section{Abstract}

Multiple indicators that affect Online Banking in China are explored in this study, namely social factors, customer preferences, and online banking factors. An artificial neural network model was performed on data that comprised of online surveys given to small business, commercial bank executives and bank customers. Our results show that attitude towards account security, understanding advantages of online banking, customer credit risk assessment and attitude of the customer towards the credit and risk information system lead to more acceptance of online banking. Neural impact direction results show that customers are more accepting of online banking if there is more account security, more convenience, lower risk for customers, and more developed credit surveillance system. While least important are phone applications, supplementary functions, and how online banking is regulated and operated.

Keywords: Online banking, artificial neural networks, China, preference customers, technological innovation.

JEL: G23 Banks, Depository Institutions, Micro Finance Institutions, Mortgages; G41 Role and Effects of Psychological, Emotional, Social, and Cognitive Factors on Decision Making in Financial Markets. 


\section{Resumo}

A inovação tecnológica e as mudanças nos estilos de vida (particularmente devido à pandemia do COVID-19) fizeram com que as interações entre consumidores e bancos mudassem subitamente. Este documento estuda múltiplos indicadores que afetam a aceitação do internet banking na China, incluindo preferência do cliente, fatores referentes ao internet banking, fatores sociais e outros. Realizaram-se questionários dirigidos a pequenos e microempresários, executivos de bancos comerciais e clientes bancários. Elaborou-se também um modelo de rede neuronal artificial com dados que compreendem mais de mil grupos. Os resultados demonstram que a atitude em relação à segurança da conta, à compreensão das vantagens do internet banking e à avaliação do risco do prestatário, além da atitude do cliente com respeito ao sistema de informação creditícia, são fatores relativamente importantes e que afetam a aceitação do internet banking. A direção de impacto de cada indicador mostra que é mais provável que os clientes aceitem o internet banking caso haja maior segurança, mais conveniência, menor risco para os clientes e um sistema de informação e vigilância de crédito mais desenvolvido. Em contrapartida, os indicadores para aplicativos de telefonia móvel, funções auxiliares, regulamentações e operações do internet banking são relativamente menos importantes.

Palavras-chave: Internet banking, rede neuronal artificial, China, preferência clientes, inovação tecnológica.

JEL: G23 Bancos, Outras instituições depositárias, Hipotecas; G41 Papel e efeitos de fatores psicológicos, emocionais, sociais e cognitivos na tomada de decisões nos mercados financeiros.

\section{Introducción}

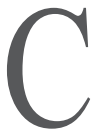

on el rápido desarrollo de la tecnología de la información moderna, el efecto de los sistemas de redes de información en la industria bancaria se ha vuelto cada vez más notable. En particular, la aplicación de pagos móviles, servicios de redes sociales, motores de búsqueda, computación en la nube, entre otras innovaciones, han acelerado la creación de nuevos modelos de negocios. La banca por internet ha surgido en respuesta a estas condiciones precisas y al tiempo madurado. Banca en línea es una institución financiera que se basa únicamente en la web para proporcionar servicios financieros, lo que permite mejorar la eficiencia del trabajo y la calidad del servicio, al tiempo que amplía su alcance y evoluciona un sistema financiero más inclusivo (Chan 2004; Chauhan, Yadav y Choudhary 2019).

En los últimos años, China ha prestado cada vez más atención al papel del internet en la promoción de oportunidades económicas y financieras, especialmente la exploración e innovación en internet y los acuerdos financie- 
ros. La Comisión Reguladora de la banca de China aprobó programas piloto en cinco bancos privados desde 2014. A pesar de las muchas ventajas de la banca por internet, su capacidad para sobrevivir y crecer depende de la aceptación de sus clientes (Laukkanen 2016; Aboobucker y Bao 2018).

Con la innovación tecnológica y los cambios en los estilos de vida, las interacciones comerciales entre los consumidores y los bancos están cambiando gradualmente de la gestión de los negocios por parte de los clientes en persona en los bancos físicos a tener acceso a los servicios financieros que necesitan directamente a través de tecnologías como la banca por internet o la banca móvil. King (2012) afirmó que la tendencia de desarrollo a futuro de los bancos será que el banco no solo será un lugar, sino una especie de comportamiento. Es decir, el modelo de negocio en el que un operador de la industria bancaria proporciona servicios a los consumidores a través de un banco físico se eliminará gradualmente, mientras que las necesidades de los consumidores de servicios financieros se satisfarán mediante servicios financieros digitales, basados en internet y dispositivos móviles (Aboobucker y Bao 2018).

En los últimos años, la tecnología financiera ha desencadenado una gran ola en todo el mundo y una innovación tan disruptiva que combina la tecnología con los servicios financieros que han supuesto una amenaza para el modelo empresarial del sector financiero tradicional (Vives 2019). En medio de esta ola, las fintech podrían reducir la dependencia de las instituciones financieras tradicionales y proporcionar servicios financieros de bajo costo y mayor valor. Sin embargo, incurrirá en los problemas que más preocupan a los consumidores, incluida la privacidad, la seguridad de la información y la protección del consumidor, si la participación y supervisión de las instituciones financieras es inadecuada (Martins, Oliveira y Popovič 2014). En vista de lo anterior, para seguir la tendencia futura y satisfacer las necesidades de los consumidores, los operadores bancarios deben realizar ajustes y mejoras en los servicios financieros existentes.

La investigación existente se ha centrado principalmente en los factores de la banca por internet que afectan a los bancos tradicionales que adoptan operaciones de internet (Al-Jabri y Sohail 2012; Boateng et al. 2016), la banca electrónica (Al-Smadi 2012; Hoehle, Scornavacca y Huff 2012; Zhang, Weng y Zhu 2018) y banca móvil (Yu 2012; Shaikh y Karjaluoto 2015). Los métodos de investigación empírica de estos casos citados se basan principalmente en modelos de regresión lineal. Teniendo en cuenta que la banca por internet se está convirtiendo en una nueva tendencia de la industria bancaria, y con el fin de 
evitar la multicolinealidad y el sobreajuste de los modelos de regresión lineal, este documento verifica sus resultados a través de enfoques teóricos alternativos. Con la ayuda de un modelo de red neuronal artificial, un modelo que se adapta a sí mismo se autoorganiza, aprende en tiempo real y es capaz de manejar información no lineal, se muestra la importancia relativa y la dirección del impacto de los factores de aceptación de internet a través de datos empíricos.

\section{Estado del arte}

La banca por internet permite que se rompan las restricciones geográficas, se amplíe el alcance de la cobertura, los servicios se vuelvan más eficientes, se reduzcan los costos de transacción y se mejore el nivel de inclusión financiera (Diniz, Porto y Adachi Nami 2005; Bojan, Mutu y Păun 2010; Sharma 2016). Sin embargo, el éxito de la banca por internet requiere de un proceso de penetración que incitaría a la producción y distribución de servicios innovadores $(\mathrm{Wu}$, Hsia y Heng 2006). Además de la realización de este fenómeno tecnológico, los clientes también tienden a tener una emoción hostil hacia la innovación (Joseph, McClure y Joseph 1999) y, por lo tanto, comprender los factores de aceptación o resistencia de los clientes hacia la innovación sería de gran importancia para el estudio de aceptación de la banca en línea (Laukannen 2016).

La actitud de los clientes hacia las nuevas tecnologías y la cognición de su valor pueden decidir o destruir su intención de comportamiento. Si la innovación brinda a los clientes experiencias difíciles al adoptarla, entonces los clientes se resistirían a ella (Heidenreich y Spieth 2013). Otro factor que influye en la aceptación de innovaciones por parte de los clientes son las experiencias pasadas que tienden a preceder al valor monetario (Zolait, Mattila y Sulaiman 2009; Welsh y Ferreira 2014). La percepción del riesgo es otro elemento significativo (Zhao et al. 2016), así como la dependencia de los productos o servicios que los clientes han formado y forjado a partir del hábito (Kleijnen, Lee y Wetzels 2009); además, los hábitos de consumo a menudo son factores importantes que impiden la aceptación de la innovación por parte del cliente. Asimismo, Welsh y Ferreira (2014) señalan que factores como el sexo, la edad y los ingresos también afectan la aceptación del cliente hacia la innovación. 
Utilizando la teoría del comportamiento planificado (TCP) y la teoría de la difusión de innovaciones (TDI), Tan y Teo (2000) descubrieron que los factores de control de comportamiento y actitudinales son los principales determinantes de la intención de adoptar la banca por internet. Basado en el modelo de aceptación tecnológica (MAT) y sus resultados empíricos, Pikkarainen et al. (2004) muestran que la utilidad percibida y la información clara fueron los principales factores de influencia en la aceptación de la banca en línea. Hanafizadeh, Keating y Khedmatgozar (2014), basados en el modelo de aceptación de tecnología extendida (TAM2) y la teoría cognitiva social (TCS), encontraron que tanto las normas subjetivas como la autoeficacia de la computadora desempeñan indirectamente un papel importante en la intención de adoptar la banca por internet. Martins, Oliveira y Popovič (2014) descompusieron la TCP y descubrieron que la adopción de la banca en línea se ve alentada por factores de actitud (características del sitio web y la utilidad percibida) e impedida por un factor de control conductual percibido (entorno externo), independientemente de las normas subjetivas. Usando MAT y algunas variables de control adicionales, Alalwan et al. (2015) encontraron que la utilidad percibida y la facilidad de uso percibida, la resistencia al cambio, la confianza, la edad, el género, la educación y los ingresos explicaron la variación en la actitud hacia la adopción de la banca en línea, mientras que las actitudes hacia el uso explicaron la mayoría de la varianza en la intención. Lee (2009) sostiene que el riesgo de seguridad, el riesgo financiero, el control del comportamiento percibido, las normas subjetivas, la actitud, los beneficios percibidos y la utilidad percibida son los determinantes importantes de la adopción de la banca por internet. El mismo autor, basado en MAT, encontró que la autoeficacia es uno de los precursores de variables como el riesgo y la experiencia en internet, y que otras condiciones facilitadoras desempeñan un papel prominente en la adopción de la banca por internet en Corea del Sur. El estudio empírico de Alalwan et al. (2015) mostró que la confianza, el conocimiento del servicio, la calidad de la producción, la diversión percibida y el diseño web contribuyeron a explicar la intención y el uso de la banca por internet. Martins, Oliveira y Popovič (2014) desarrollaron un modelo conceptual para explicar la intención de comportamiento y el comportamiento de uso. Sus hallazgos mostraron que la expectativa de rendimiento, la expectativa de esfuerzo, la influencia social y el papel del riesgo fueron fuertes predictores de intención. Laukkanen (2016) señaló que el uso, el valor, el riesgo, la 
tradición, la imagen, el género, la edad y los ingresos fueron los principales factores que afectaron la aceptación del cliente de la banca por internet. Los resultados empíricos basados en los modelos de logit muestran que el factor de valor es el inhibidor más fuerte de la adopción de la banca por internet, y que el factor tradicional explica el rechazo de la banca por internet.

Las teorías existentes y los estudios empíricos sobre la aceptación de la banca por internet se basan principalmente en la asunción de decisiones racionales de los consumidores. Sin embargo, en muchos casos, las decisiones de los clientes se toman de acuerdo con sus sentimientos, emociones, instintos o experiencia asumida, lo cual no es un comportamiento racional en su sentido completo (Zhao et al. 2016). Los factores sociales y culturales también pueden afectar la aceptación de la banca por internet. Se deben considerar factores sociales, políticos, económicos, culturales y tecnológicos para investigar la aceptación de la banca por internet. Por lo tanto, se necesita un nuevo método en el caso de muestras limitadas e indicadores múltiples. Para compensar estas deficiencias, este trabajo utiliza métodos de redes neuronales artificiales aplicados a una encuesta multidimensional para estudiar los factores de aceptación de la banca en línea en China.

\section{Metodología}

\section{Selección de indicadores}

Según la literatura existente, se dividen los factores que afectan la aceptación del cliente de la banca por internet en tres categorías: factores del cliente, factores de la banca por internet y factores sociales.

1. Factores del cliente. Se puede dividir en dos aspectos: comportamiento y hábito del cliente, y percepción del riesgo del cliente. Con respecto al comportamiento del cliente, el primero pregunta si los clientes reconocen las ventajas de la banca por internet. Como la banca por internet difiere mucho de los bancos tradicionales, si los clientes no tienen claras las ventajas y desventajas de la banca por internet, su intención de aceptar la banca en línea se reducirá. Otro factor es si el cliente tiene un sentido de excepcionalidad hacia la banca por internet. Esto se refiere a la percepción de superioridad incurrida 
cuando el cliente acepta los productos o servicios de la banca en línea. Si los clientes pudieran fortalecer su autoimagen y obtener una sensación de excepcionalidad mediante el uso de la banca por internet, entonces los clientes tendrían más probabilidades de aceptarla (Gerrard, Cunningham y Devlin 2006). El tercero es si el cliente aboga por el cambio. La actitud de un cliente hacia el cambio determinaría si el cliente se ha formado un estereotipo sobre los bancos tradicionales y se ha vuelto dependiente de él. El cuarto son las habilidades de operación de internet. La capacidad de un cliente para operar computadoras y usar dispositivos móviles de internet afectaría su aceptación hacia la banca por internet. El quinto son las características personales, los rasgos personales como la edad, el sexo, el nivel educativo y el nivel de ingresos, que también afectarían esta aceptación.

Con respecto a la percepción de riesgo del cliente, el primer elemento es la preferencia de riesgo del cliente. La teoría del mercado financiero señala que los participantes financieros se dividen en tres categorías: amante del riesgo, aversión al riesgo y neutral al riesgo. El segundo elemento es la conciencia del cliente sobre la banca por internet. Un banco de internet puro (que no existe de manera física) es una institución que obtiene una licencia legal emitida por las autoridades reguladoras y acepta su estricta supervisión. La mayor diferencia entre los bancos de internet y los bancos tradicionales es la transformación del modo de servicio. Además de los riesgos operativos, de reputación y de crédito que enfrentan todas las instituciones bancarias, el riesgo de la banca por internet incluye riesgos de identificación remota, almacenamiento de datos y protección de la privacidad y riesgos de seguridad de datos. La forma en que un cliente ve el riesgo del nuevo modo de servicio tendría un impacto directo en la aceptación de la banca por internet.

2. Factores de la banca por internet. El primer factor que afecta la aceptación de la banca por internet es la calidad de la conexión a las redes electrónicas, ya que las operaciones de banca por internet dependen únicamente de la conexión para proporcionar servicios a una gran cantidad de clientes actuales y potenciales. El segundo es su conveniencia, que se refiere a la operación simple, el ahorro de tiempo y costos, y la eficiencia obtenida al participar en transacciones en la plataforma de banca por internet. El tercero es la confiabilidad, es decir, el tema de seguridad, principalmente enfocándose en la seguridad de la cuenta y la seguridad de la privacidad. La principal preocupación del cliente es la seguridad de la cuenta, ya que los 
sistemas de información pueden ser pirateados o violados por phishing u otro software. El cuarto elemento son las funciones auxiliares, que se refieren a otros servicios prestados además de los préstamos normales; por ejemplo, medios interactivos como video, audio y realidad virtual. El aumento de las funciones auxiliares podría mejorar potencialmente la calidad del servicio y la satisfacción de la experiencia del cliente, y así mejorar la aceptación del cliente de la banca por internet.

3. Factores sociales. La actitud de un cliente hacia el entorno social de la banca por internet también puede afectar el grado de aceptación. Primero está la actitud hacia todo el sistema de crédito; la banca por internet se lleva a cabo sin interacciones personales y su desarrollo exitoso depende del estado crediticio de todo el sistema. Si los clientes creen que el sistema de crédito de China no es líquido y no puede cubrir las pérdidas de préstamos de la banca por internet, entonces los clientes la rechazarían. Por lo tanto, la expectativa de los clientes de la banca por internet está estrechamente vinculada a la percepción de un sistema de crédito saludable.

El segundo factor es la actitud de los clientes hacia el sistema de información crediticia. Un mejor sistema de información crediticia es fundamental para que los bancos determinen las aprobaciones de préstamos, y es una piedra angular en el desarrollo de un sistema de información crediticia multinivel, multidimensional y diversificado.

Un tercer factor para la aceptación de la banca por internet son las regulaciones legales en el desarrollo de la industria de la banca por internet. La industria bancaria siempre ha sido cuidadosamente regulada y supervisada; por lo tanto, el auge de la banca por internet crea un entorno legal más vigilante y regulatorio.

El cuarto factor que se estudia es la relación entre la banca por internet y la banca tradicional. Según este análisis, el público tiene tres tipos de opiniones sobre esta relación: competitiva, complementaria y una relación competitiva y cooperativa mixta. La expectativa del cliente de la banca por internet varía según estas opiniones diferentes y está relacionada con la infancia de la industria de la banca por internet. Una relación competitiva resultaría despiadada y fatal, sin embargo, una relación complementaria permitiría a la banca por internet crear un nicho de mercado, al tiempo que le permitiría cooperar con la banca tradicional en ciertos aspectos. 


\section{Descripción y análisis de datos}

De acuerdo con el análisis teórico presentado, existen 15 factores que afectan la aceptación de la banca por internet (tabla 1). Los factores que afectan la aceptación de la banca por internet se estudian a través de cuestionarios en línea. Los cuestionarios fueron realizados por los autores y distribuidos a los propietarios de pequeñas y microempresas, empleadores de bancos comerciales y al público en general en 2017, en el ámbito nacional en China. Los cuestionarios fueron enviados a través de redes universitarias y con la cooperación de bancos comerciales que contenían su propia base de datos de clientes. El cuestionario diseñado cubre los diversos factores mencionados en la sección anterior. Se consultó la experiencia pasada y la experiencia de muchos expertos en campos relacionados para diseñar la encuesta en línea. Estos incluyen ejecutivos bancarios, profesionales de finanzas de internet, profesores universitarios y autoridades reguladoras gubernamentales. Se establecieron un total de 27 preguntas con 16 indicadores. Se recolectó un total de 1634 cuestionarios, excluyendo cuestionarios incompletos y muestras más allá del alcance de las medianas, pequeñas y microempresas, se seleccionaron 1136 cuestionarios válidos, que alcanzó una tasa efectiva del $70 \%$.

Las estadísticas descriptivas se enumeran en la tabla 1. El método de medición de escala de 6 puntos se utilizó para todas las preguntas con selecciones de escala. Por ejemplo, hay dos preguntas sobre la excepcionalidad de la banca por internet: “¿entiende las ventajas de la banca por internet?”, y “¿entiende las desventajas de la banca por internet?". La escala del 1 al 6 se clasifica así: "completamente sin comprensión, básicamente sin comprensión, un poco confuso, un poco de comprensión, comprensión básica, comprensión completa".

El puntaje promedio para estas preguntas es 3,82, lo que indica que la mayoría de los clientes encuestados comprenden las ventajas y desventajas de la banca por internet. Este resultado es promovido por la fusión continua de las operaciones bancarias con las tecnologías de internet en las últimas décadas y el rápido desarrollo de las finanzas de internet en años recientes.

Una desviación estándar de 1,54 muestra que el desacuerdo entre los clientes no es muy obvio y es relativamente consistente. Se aplicó una escala 
de 3 a 6 puntos para algunos cuestionarios, y la confiabilidad de la escala fue verificada por el alfa de Cronbach. ${ }^{1}$

La tabla 1 muestra que el alfa de Cronbach para esta investigación está entre 0,68 y 0,88 , lo que sugiere una alta confiabilidad.

Tabla 1

Análisis de las estadísticas descriptivas y de confiabilidad

\begin{tabular}{|c|c|c|c|c|c|c|}
\hline Factores & Variables & $\begin{array}{c}\text { Número de } \\
\text { asuntos }\end{array}$ & $\begin{array}{l}\text { Indicadores } \\
\text { secundarios }\end{array}$ & Media & $\begin{array}{l}\text { Desviación } \\
\text { estándar }\end{array}$ & $\begin{array}{l}\text { Alfa de } \\
\text { Cronbach }\end{array}$ \\
\hline \multirow{7}{*}{$\begin{array}{l}\text { Factores } \\
\text { clientes }\end{array}$} & \begin{tabular}{|l} 
Ventajas de \\
banca en línea
\end{tabular} & 2 & \begin{tabular}{|l|} 
Ventajas y \\
desventajas
\end{tabular} & 3,82 & 1,54 & 0,73 \\
\hline & \begin{tabular}{|l} 
Sentido de \\
reconocimiento
\end{tabular} & 1 & --- & 3,06 & 1,88 & 0,69 \\
\hline & Avoca el cambio & 1 & --- & 2,87 & 2,02 & 0,75 \\
\hline & $\begin{array}{l}\text { Habilidades de } \\
\text { internet }\end{array}$ & 2 & $\begin{array}{l}\text { Computadora y } \\
\text { dispositivo móvil }\end{array}$ & 4,61 & 0,81 & 0,88 \\
\hline & $\begin{array}{l}\text { Características } \\
\text { personales }\end{array}$ & 4 & $\begin{array}{l}\text { Sexo, edad, } \\
\text { educación, ingreso }\end{array}$ & 2,12 & 1,13 & 0,82 \\
\hline & $\begin{array}{l}\text { Tolerancia de } \\
\text { riesgo }\end{array}$ & 1 & |---- & 3,97 & 1,58 & 0,79 \\
\hline & Perfil de riesgo & 2 & $\begin{array}{l}\text { Prestador } \\
\text { prestatario }\end{array}$ & 3,01 & 2,22 & 0,77 \\
\hline \multirow{4}{*}{$\begin{array}{c}\text { Factores } \\
\text { banca } \\
\text { internet }\end{array}$} & \begin{tabular}{|l} 
Calidad \\
de conexión
\end{tabular} & 1 & --- & 4,83 & 0,79 & 0,86 \\
\hline & Conveniencia & 3 & $\begin{array}{l}\text { Operación, costo, } \\
\text { eficiencia }\end{array}$ & 4,18 & 1,24 & 0,82 \\
\hline & Confiabilidad & 2 & $\begin{array}{l}\text { Seguridad, } \\
\text { privacidad }\end{array}$ & 2,87 & 1,95 & 0,77 \\
\hline & $\begin{array}{l}\text { Funciones } \\
\text { auxiliares }\end{array}$ & 1 & --- & 2,99 & 1,33 & 0,7 \\
\hline
\end{tabular}

1. El alfa de Cronbach es una forma efectiva de medir la confiabilidad, que resuelve las deficiencias del método binario y a menudo se usa en el análisis de confiabilidad. Generalmente se reconoce que un alfa de Cronbach superior a 0,6 se refiere a una alta confiabilidad, y un alfa de Cronbach menor a 0,35 se refiere a bajo confiabilidad. 


\begin{tabular}{|c|l|c|l|c|c|c|}
\hline Factores & Variables & $\begin{array}{c}\text { Número de } \\
\text { asuntos }\end{array}$ & \multicolumn{1}{|c|}{$\begin{array}{c}\text { Indicadores } \\
\text { secundarios }\end{array}$} & Media & $\begin{array}{c}\text { Desviación } \\
\text { estándar }\end{array}$ & $\begin{array}{c}\text { Alfa de } \\
\text { Cronbach }\end{array}$ \\
\hline \multirow{2}{*}{$\begin{array}{c}\text { Factores } \\
\text { socio- } \\
\text { ambien- } \\
\text { tales }\end{array}$} & $\begin{array}{l}\text { Estado del } \\
\text { rédito social }\end{array}$ & 1 & --- & 3,86 & 1,21 & 0,81 \\
\cline { 2 - 7 } & $\begin{array}{l}\text { Ambiente de ley } \\
\text { y regulación }\end{array}$ & 2 & $\begin{array}{l}\text { Leyes, } \\
\text { regulaciones }\end{array}$ & 3,55 & 1,45 & 0,73 \\
\cline { 2 - 7 } \\
\cline { 2 - 7 } \\
\cline { 2 - 7 } & $\begin{array}{l}\text { Sempetitivas } \\
\text { crédito personal de }\end{array}$ & 3 & $\begin{array}{l}\text { Competencia, } \\
\text { cooperación, } \\
\text { complementariedad }\end{array}$ & 3,62 & 1,58 & 0,75 \\
\hline
\end{tabular}

Fuente y elaboración propia.

La tabla 2 presenta la descripción estadística de la variable dependiente, en donde el 42,8\% de las 1136 observaciones no aceptaban los productos y servicios de la banca por internet, mientras que el número de personas con una predisposición positiva representa el $57,2 \%$, lo que sugiere que el mercado tiene expectativas sobre la banca por internet.

Tabla 2

Descripción estadística de la variable dependiente

\begin{tabular}{|l|l|c|c|}
\hline \multicolumn{1}{|c|}{ Variable dependiente } & \multicolumn{1}{|c|}{ Opciones } & Monto & Probabilidad \\
\hline \multirow{2}{*}{$\begin{array}{l}\text { ¿Está dispuesto a aceptar } \\
\text { productos y servicios ofrecidos } \\
\text { por la banca por internet? }\end{array}$} & Reacio & 486 & $42,8 \%$ \\
\cline { 2 - 4 } & Complaciente & 650 & $57,2 \%$ \\
\hline
\end{tabular}

Fuente y elaboración propia. 


\section{Modelo de investigación}

Se realizó un análisis de los indicadores de información del cliente que reflejan la importancia de la aceptación de la banca por internet con la ayuda del modelo de red neuronal artificial (RNA). Tradicionalmente, la correlación de variables independientes y variables dependientes para datos discretos se realiza a través de modelos lineales y logarítmicos. Dado que los datos de este documento se derivaron de cuestionarios, y la mayoría de estos datos son altamente subjetivos, la relación entre variables independientes y variables dependientes no es necesariamente lineal, por lo tanto, fue más adecuado elegir modelos lineales y no lineales para analizar datos. Además, se analizaron 29 variables, por lo que los coeficientes que debían estimarse incrementaron exponencialmente con el aumento de las variables. Después de una consideración exhaustiva, se seleccionó el modelo de RNA para examinar los factores que afectan la aceptación de la banca por internet. El proceso empírico sigue el siguiente procedimiento:

\section{Construir una red neuronal $\rightarrow$ Calcular la importancia relativa de cada nodo $\rightarrow$ Estimar la dirección de respuesta de aceptación de la banca por internet contra cada indicador.}

Las RNA son una familia de modelos matemáticos utilizados para el procesamiento de información, inspirados por las conexiones sinápticas del cerebro humano; son modelos informáticos que consisten en una gran cantidad de nodos (o neuronas) conectados entre sí (Hornik 1993). Cada nodo representa una función de salida específica, que se denomina función de activación. La conexión entre dos nodos representa un valor ponderado para la señal de conexión y es equivalente a la memoria de la red neuronal artificial. La salida de la red depende del valor de peso, la función de incentivo y las diferentes formas en que la red está conectada. La red en sí misma suele ser una aproximación a un algoritmo o la expresión de una estrategia lógica. Hornik (1993) demuestra que después de un entrenamiento suficiente, una RNA de tres capas, con suficientes nodos de capa oculta, puede aproximarse a cualquier función no lineal con precisión. Como resultado, se diseñó una red neuronal artificial de tres capas para este artículo. 
Gráfico 1

Topología general de un modelo de RNA para la evaluación de indicadores de aceptación de banca por internet

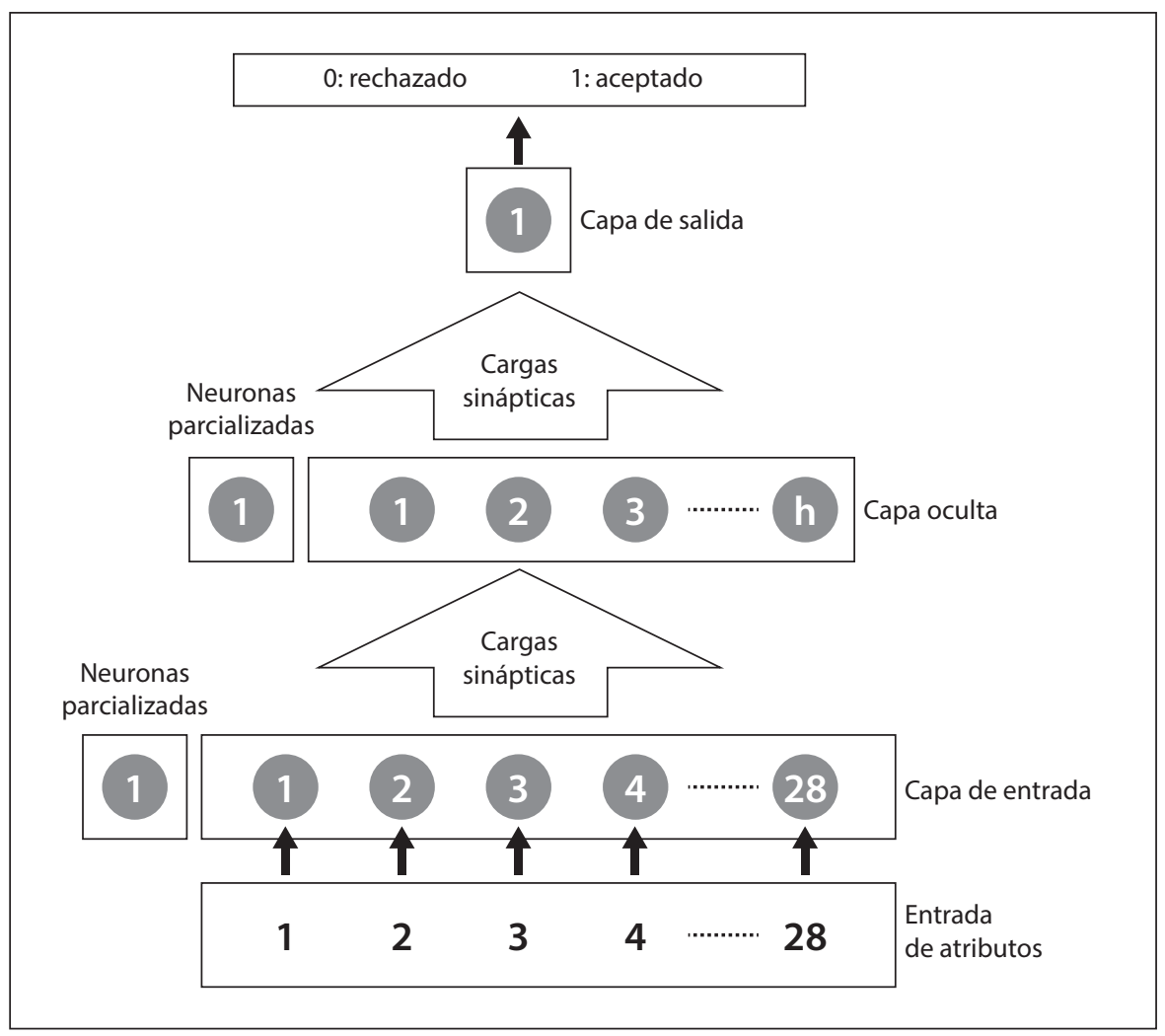

Elaboración propia.

El gráfico 1 ilustra una estructura de red neuronal de tres capas con una capa de entrada, una capa oculta y una capa de salida. Las neuronas de cada capa están conectadas a todos los nodos de la capa adyacente, y cada articulación tiene un peso sináptico para ajustar los valores de enlace. Se establecen dos capas de polarización en la capa de entrada y la capa oculta, cada una apuntando a los nodos de la capa oculta y los nodos de la capa de salida, respectivamente. La capa de polarización puede considerarse como una tenden- 
cia activa del sensor sin relación con su entrada. El proceso de aprendizaje de la red neuronal consiste en modificar el peso sináptico y el sesgo. El nodo de la capa de entrada se expresa como $I_{1}, I_{2}, I_{3} \ldots, I_{28}$, que representan los 28 indicadores de factores que afectan la aceptación, respectivamente; el nodo de la capa oculta se expresa como $H_{1}, H_{2}, H_{3} \ldots H_{h}$, mientras que $W_{i j}$ expresa el peso de la conexión entre $I_{\mathrm{i}}$ y $\mathrm{H}_{\mathrm{j}}$, y el valor del nodo de la capa oculta se puede expresar como:

$$
H_{j}=T F\left(\sum_{i=1}^{h} W_{i j} \times I_{i}\right)
$$

De igual manera, el valor de la capa de salida $\mathrm{O}_{k}$ para la red neuronal se puede expresar de la siguiente manera:

$$
O_{k}=T F\left(\sum_{i=1}^{28} W_{j k} \times H_{i}\right)
$$

En donde:

$T F$ es una función de transferencia no-lineal que captura la relación no-lineal entre los nodos de entrada y salida. Este trabajo utiliza una función de lógica sigmoide para realizar la clasificación que se expresa como:

$$
f(x)=1 /\left(1+e^{-u}\right)
$$

Esta función de transferencia permite convertir de una red neuronal lineal a una no lineal. El valor del nodo de entrada asignado por la red neuronal es determinado por la importancia de la información que carga la neurona, y el valor de la capa oculta representa la contribución a la capa de salida. Consecuentemente, la importancia del nodo de entrada puede ser calculada en función del nivel de afectación de algunos nodos. El método Garson (1991) se aplicó para calcular el valor de contribución de los nodos de entrada. El algoritmo de Garson usa el producto del peso de las conexiones para calcular el grado de contribución de los nodos ocultos $j$ a los nodos de salida $o$. La contribución de cada nodo de entrada $k$ contra cada nodo oculto $j$ se puede expresar así: 


$$
r_{i j o}=\left|w_{i j} \times w_{j o}\right| /\left(\sum_{k=1}^{28}\left|w_{i j} \times w_{j o}\right|\right)
$$

Por la tanto, la contribución total del nodo de entrada es:

$$
T_{i}=\sum_{j=1}^{h} r_{i j o}
$$

Entonces, la importancia relativa del nodo de entrada $i$ se puede calcular:

$$
R I_{i}=\left(T_{i} / \sum_{k=1}^{28} T_{k}\right)
$$

Se seleccionó el algoritmo de aprendizaje de red neuronal de retropropagación más utilizado, y su proceso de cálculo del modelo de red de tres capas es:

1. Se selecciona una matriz de peso de conexión al azar de la capa de entrada a la capa oculta, y de la capa oculta a la capa de salida para establecer el error de convergencia general.

2. Se realiza un proceso supervisado de aprendizaje y capacitación en la red de acuerdo con la muestra de la matriz de aprendizaje. Se calcula el error de la salida real y la salida esperada de la red, y se adopta el algoritmo de aprendizaje de propagación de error de retroceso para ajustar los pesos de conexión de la capa de entrada a la capa oculta, y de la capa oculta a la capa de salida.

3. Cuando el error de convergencia de la salida del modelo es mayor que el error establecido, se regresa al paso 2 o se finaliza el entrenamiento y se calcula el valor predictor de acuerdo con el peso de conexión y el valor umbral a través de la fórmula 1. Cabe señalar que el aprendizaje supervisado y la capacitación establece una función de error como el valor de referencia del error de convergencia, y su fórmula es la siguiente:

$$
E=\frac{1}{2} \sum_{l=1}^{L} \sum_{h=1}^{H}\left(o_{l h}-y_{l h}\right)^{2}
$$

Donde:

$l=1,2,3 \ldots, L$ es el valor observado de entrada y salida; $h=1,2,3 \ldots$, $H$ es el nodo salida; $o_{l h}$ es el valor predicho de salida, y $y_{l h}$ es la salida real. 


\section{Resultados}

\section{Calibración del modelo}

Para construir un modelo óptimo de red neuronal se utilizó el método de búsqueda pulgada por pulgada para identificar nodos de capa oculta, es decir, aumentando el número de nodos de capa oculta de 0 y tomando el error de red total como el estándar para juzgar el modelo óptimo de red neuronal. Este error total es la suma del error de entrenamiento y el error de predicción. Otro factor que afecta el resultado del modelo son los diferentes coeficientes de aprendizaje y las diferentes proporciones de entrenamiento/datos pronosticados (tabla 3).

Después de realizar una gran cantidad de pruebas y comparaciones repetidas se tabularon los parámetros correspondientes y se seleccionó el error total de los modelos más óptimos, incluidos los que tienen diferentes parámetros de aprendizaje y distribuciones de entrenamiento/prueba, y se determinó que los modelos que mejor se ajustan tienen 28 nodos de entrada, 1 capa oculta, 8 o 10 capas neuronales ocultas, 1 capa de salida, y todos los modelos tienen su función inicial establecida aleatoriamente con el valor del parámetro inicial de [-0,3: 0,3]. Además, los modelos elegidos incluyen una función actualizable que representa su estructura topológica, una función de activación de características logarítmicas, una iteración máxima de 2000 veces y un error total que prueba la validez de los modelos. Se observó que el modelo con un factor de aprendizaje de 0,0079 y una distribución de entrenamiento / prueba de 50-50 tenía una ligera ventaja y, en consecuencia, fue elegido como el modelo que mejor se ajustaba a nuestros propósitos.

El gráfico 2 indica el error de entrenamiento de la red neuronal y la tasa predicha de los nodos de varias capas ocultas, sus factores de aprendizaje, distribuciones de entrenamiento/prueba y otros parámetros consistentes. Cuando el número del nodo de capa oculta es 1, la red neuronal es equivalente a un modelo de registro lineal. Sin embargo, al aumentar el número de nodos de capa ocultos, el error de entrenamiento del modelo se redujo gradualmente, mientras que la precisión de la predicción también mejoró gradualmente. Cuando el número de nodos de capa ocultos excede a 10, el error de entrenamiento comienza a aumentar y la precisión de la predicción 
Tabla 3

\section{Parámetros de entrenamiento para redes neuronales y error}

\begin{tabular}{|l|c|c|c|c|c|c|}
\hline \multicolumn{1}{|c|}{ Modelos } & $\mathbf{1}$ & $\mathbf{2}$ & $\mathbf{3}$ & $\mathbf{4}$ & $\mathbf{5}$ & $\mathbf{6}$ \\
\hline $\begin{array}{l}\text { Capa de nodos de } \\
\text { entrada }\end{array}$ & 28 & 28 & 28 & 28 & 28 & 28 \\
\hline Capa de nodos ocultos & 8 & 10 & 8 & 10 & 8 & 10 \\
\hline $\begin{array}{l}\text { Capa de nodos } \\
\text { de salida }\end{array}$ & 1 & 1 & 1 & 1 & 1 & 1 \\
\hline Función inicial & Aleatoria & Aleatoria & Aleatoria & Aleatoria & Aleatoria & Aleatoria \\
\hline Función de aprendizaje & $\mathrm{BP}$ & $\mathrm{BP}$ & $\mathrm{BP}$ & $\mathrm{BP}$ & $\mathrm{BP}$ & $\mathrm{BP}$ \\
\hline $\begin{array}{l}\text { Coeficiente } \\
\text { de aprendizaje }\end{array}$ & 0,0079 & 0,0079 & 0,0081 & 0,0081 & 0,0095 & 0,0095 \\
\hline $\begin{array}{l}\text { Rango de carga } \\
\text { inicial aleatoria }\end{array}$ & {$[-0,30,3]$} & {$[-0,30,3]$} & {$[-0,30,3]$} & {$[-0,30,3]$} & {$[-0,30,3]$} & {$[-0,30,3]$} \\
\hline $\begin{array}{l}\text { Función de } \\
\text { actualización }\end{array}$ & Topológica & Topológica & Topológica & Topológica & Topológica & Topológica \\
\hline \begin{tabular}{l} 
Función de activación \\
\hline $\begin{array}{l}\text { Iteraciones máximas } \\
\text { permitidas }\end{array}$
\end{tabular} & Logística & Logística & Logística & Logística & Logística & Logística \\
\hline $\begin{array}{l}\text { Capacitación/ } \\
\text { predicción proporcional } \\
\text { de datos (\%) }\end{array}$ & $50 / 50$ & $50 / 50$ & $50 / 50$ & $50 / 50$ & $50 / 50$ & $50 / 50$ \\
\hline Error total & 13,1 & 12,1 & 12,9 & 12,6 & 13,2 & 12,7 \\
\hline
\end{tabular}

Elaboración propia.

disminuye. En conclusión, el modelo de red neuronal, presentado en este artículo, con 10 nodos de capa ocultos y una reiteración de 2000 veces tiene la combinación dual más óptima de error de entrenamiento más bajo y precisión de predicción más alta. Esto sugiere que una RNA de tres capas construida bajo las condiciones mencionadas es capaz de aproximar la relación no lineal entre los indicadores del cliente y la aceptación de la banca por internet con mayor precisión. 


\section{Gráfico 2}

\section{Error total y predicción óptima de la precisión del modelo de redes neuronales}

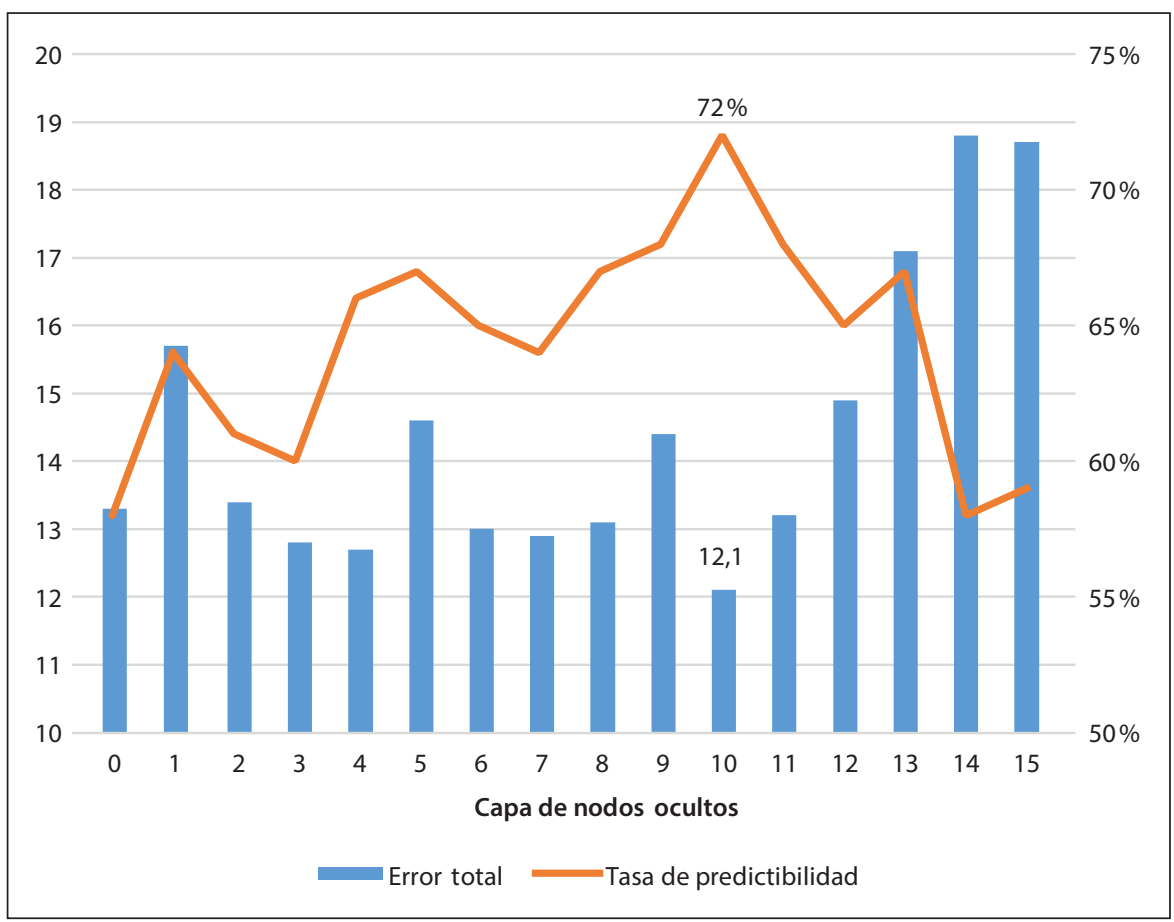

Elaboración propia.

\section{Resultados y análisis de prueba empírica}

\section{Dirección de la neurona de impacto}

La dirección del efecto de cada neurona contra la variable dependiente se juzga por el signo del peso sináptico de cada neurona. Los resultados se muestran en la tabla 4. Si una neurona tiene un signo positivo "+" al lado de, por ejemplo, "operación”, indica que el modelo de negocio de la banca por internet es relativamente fácil de operar y, por lo tanto, existe un mayor grado de aceptación de la banca por internet. El signo de las neuronas "sexo", 
"edad" y "nivel educativo" es negativo “-”, lo que indica que las mujeres, los clientes de mayor edad y los clientes de educación superior tienden a no aceptar la banca por internet. La razón es que las usuarias mayores y las usuarias con niveles de educación superior son más cautelosas, reacias a la novedad y necesitan un proceso de aceptación relativamente largo. Las neuronas "riesgo de prestamista" y "riesgo de prestatario" también son negativos “-", lo que indica que cuanto mayor es el reconocimiento del riesgo de la banca por internet, menor es el grado de aceptación. Además, la dirección de respuesta de otras neuronas frente a las variables dependientes es positiva, lo que sugiere que cuanto mayor sean los valores de estas variables, mayor será el grado de aceptación del usuario de la banca por internet. Por ejemplo, cuanto mayor es la preferencia por el riesgo, más segura es la cuenta y mayor será el grado de aceptación de la banca por internet.

Tabla 4

Dirección de impacto neuronal para la aceptación de banca por internet

\begin{tabular}{|l|c|l|c|}
\hline \multicolumn{1}{|c|}{ Neurona } & Sentido & \multicolumn{1}{c|}{ Neurona } & Sentido \\
\hline Calidad del internet & + & Cambio & + \\
\hline Función auxiliar & + & Edad & - \\
\hline Regulación & + & Educación & - \\
\hline Habilidades computacionales & + & Competencia & - \\
\hline Habilidad dispositivo móvil & + & Eficiencia & + \\
\hline Género & - & Complementariedad & + \\
\hline Leyes & + & Desventajas & + \\
\hline Operación & + & Privacidad & + \\
\hline Riesgo prestador & - & Riesgo & + \\
\hline Crédito social & + & Sistema crédito vigilancia & + \\
\hline Cooperación & + & Sistema de información crédito & + \\
\hline Ingreso & + & Riesgo prestatario & - \\
\hline Seguridad & + & Ventaja & + \\
\hline Concienciación & + & Costo & + \\
\hline
\end{tabular}

Elaboración propia. 


\section{Análisis de importancia relativa}

Después de determinar la estructura de la red y los pesos del modelo de aceptación de la banca por internet, la importancia relativa de cada nodo de entrada se puede calcular de acuerdo con el algoritmo de Garson, como se muestra en la ecuación (3). Los resultados se resumen en el gráfico 3. El más destacado de los 28 nodos de entrada es la seguridad de la cuenta, con una importancia relativa cercana al 16,4\%, es decir, la seguridad de la cuenta es el elemento más pesado para decidir si los clientes aceptan productos o servicios de banca por internet. Dado que la banca por internet no tiene un lugar físico y las actividades financieras no se realizan cara a cara, las vulnerabilidades de la red y los ataques de virus pueden crear incertidumbre y riesgo para los usuarios de la banca por internet.

Otro indicador relativamente importante son las ventajas de utilizar los servicios de banca por internet. Según los resultados, las ventajas tienen una importancia del $12,1 \%$, lo que destaca que la aceptación del cliente de la banca por internet se debe a su conveniencia, tarifas de transacción reducidas y confirmación instantánea. Además, la eficiencia mejorada de las actividades financieras permite un mayor alcance de las actividades financieras y compensa los segmentos del mercado que los bancos tradicionales no pueden atender.

El siguiente factor importante de aceptación es el riesgo del prestatario con una importancia relativa del 9,5\%. La banca en línea emplea grandes bases de datos para realizar consultas de verificación de crédito con el fin de aprobar préstamos o servicios financieros para clientes de activos livianos. En caso de incumplimiento, todas las pérdidas correrían a cargo del banco de internet. Además, un banco de internet suele ser un banco privado de pequeña escala que no puede hacer cumplir un sistema rígido de pago, ni esperar rescates del gobierno cuando falla. Los clientes, por lo tanto, consideran que el riesgo del prestatario está directamente relacionado con la supervivencia de la banca por internet.

El desarrollo de un sistema de información crediticia y un sistema de vigilancia crediticia también determina la aceptación del cliente de la banca por internet en gran medida, con una importancia relativa de 8,6 y 7,0 \% respectivamente. El estado crediticio del prestatario puede evaluarse desde varios aspectos de acuerdo con la multidimensionalidad de big data. Sin embargo, los sistemas de información y vigilancia de crédito de China están principalmente bajo la operación del gobierno, y los datos en poder del gobierno son solo la 
punta del iceberg en comparación con los grandes datos. El potencial de los grandes datos necesita ser explotado para obtener el valor real de un sistema de vigilancia crediticia. Por lo tanto, la actitud de los clientes hacia el sistema de información crediticia y el sistema de vigilancia crediticia del gobierno se convierte en un factor determinante para aceptar la banca por internet.

El riesgo del cliente también se ha convertido en una razón esencial para aceptar la banca por internet, cuya importancia relativa es del 5,9\% en el modelo presentado. En circunstancias normales, el alto riesgo se acompaña de un alto rendimiento. Los clientes con alta tolerancia al riesgo tienden a aceptar la banca por internet. Además, la percepción de privacidad del cliente y el nivel de protección de esta privacidad determina la aceptación del cliente de la banca por internet. Dado que los bancos de internet recopilan y almacenan una gran cantidad de datos de los clientes, la filtración o el robo de esta información confidencial podrían causar un daño grave al interés de los clientes. La importancia relativa de "si los bancos de internet pueden proteger la privacidad de los clientes", "si los clientes conocen la banca por internet" y "si los clientes entienden las desventajas de la banca por internet" es de 5,0, 4,8 y 4,6\%, respectivamente.

Entre todos los indicadores, los factores más irrelevantes, según el modelo, son las habilidades móviles, las funciones auxiliares, la regulación y la operación, cada una con una importancia relativa de menos del $1 \%$.

\section{Conclusiones}

Este documento intentó llenar un vacío en la literatura existente sobre los indicadores de aceptación de la banca por internet y el sobreajuste de los métodos de regresión lineal mediante la aplicación de un modelo de red neuronal artificial en una encuesta multidimensional en China. El estudio empírico revela la dirección del impacto y la importancia relativa entre cada indicador y la aceptación de la banca por internet. Se determinó que es más probable que los clientes acepten la banca por internet si los productos y servicios son más seguros, si puede proporcionar más ventajas, si el riesgo del cliente es bajo, si la información crediticia y el sistema de vigilancia están más desarrollados, y si los clientes tienen una mayor tolerancia al riesgo de 
Gráfico 3

La importancia relativa de cada nodo de entrada a nodo de salida

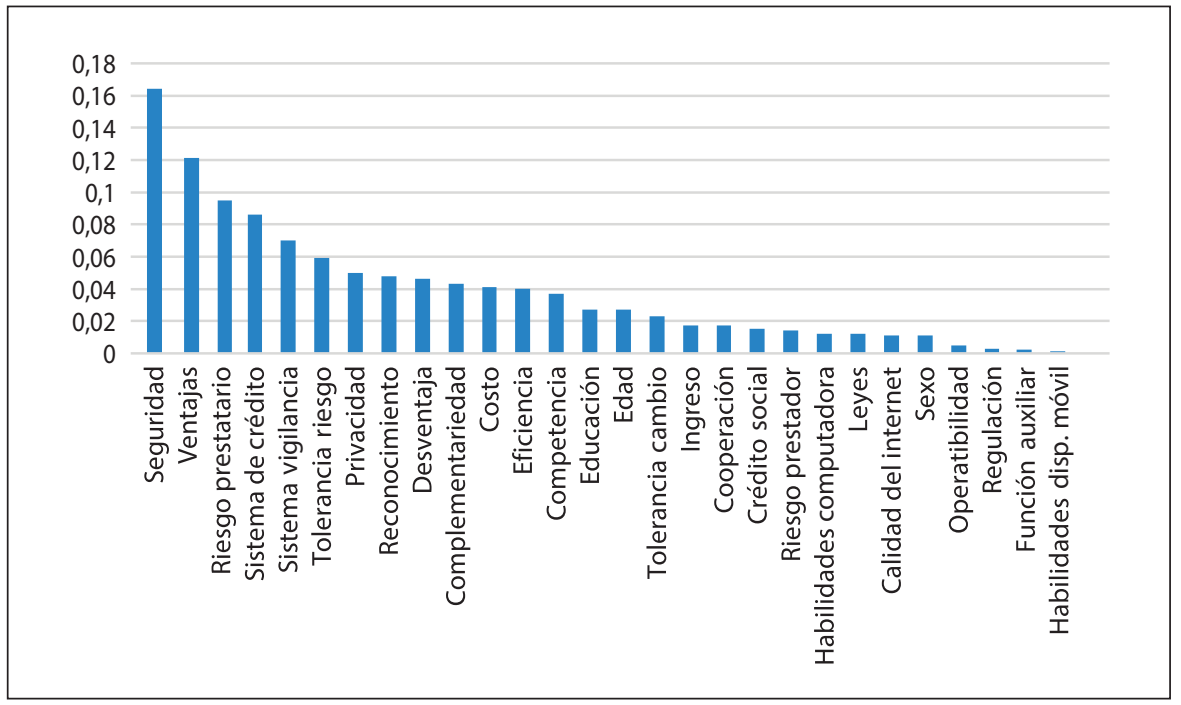

Elaboración propia.

crédito. En contraste, los indicadores con menor relevancia para la aceptación de la banca por internet en este estudio fueron: habilidades de telefonía móvil, funciones complementarias, y la regulación y operación de la banca por internet. Estos últimos resultados pueden reflejar el hecho de que el uso de dispositivos electrónicos y dinero electrónico son prevalentes en China, $\mathrm{y}$ las regulaciones gubernamentales son fuertes y generalmente acatadas, por lo que cobrarían menos relevancia.

Con la creciente demanda de productos y servicios financieros en el mundo, particularmente por el confinamiento obligatorio debido a la última pandemia, el desarrollo potencial de la banca por internet es inconmensurable. Este desarrollo no está exento de desafíos: adopción tardía de la banca por internet, políticas y regulaciones contradictorias y en conflicto, varios niveles de conocimiento y comprensión de la banca en línea, posición y segmentación inciertas en el mercado y distintos niveles de habilidades técnicas del usuario. Sin embargo, este estudio ha podido identificar los fac- 
tores importantes que deben desarrollarse para formular una estrategia para aumentar la aceptación de la banca por internet: un excelente sistema de seguridad y soporte técnico; el fomento de más comunicación e intercambio con el mercado, sensibilización a los clientes sobre los objetivos de la banca por internet, ayuda a la comprensión de la banca por internet; identificación precisa de grupos de clientes; formación de ventajas competitivas centrales; desarrollo de un sistema de información y vigilancia crediticia que cree un sistema efectivo de evaluación crediticia; y creación de leyes y regulaciones relevantes. La metodología y los resultados realizados en este estudio podrían servir a otras regiones geográficas donde los patrones y las conductas del servicio de banco por internet son distintas $\mathrm{y}$, por ende puede llevar a diferentes conclusiones.

\section{Referencias}

Aboobucker, Ilmudeen, y Yukun Bao. 2018. "What Obstruct Customer Acceptance of Internet Banking? Security and Privacy, Risk, Trust and Website Usability and The Role of Moderators". The Journal of High Technology Management Research 29 (1): 109-123. 10.1016/j.hitech.2018.04.010.

Alalwan, Alí Alalwan, Dwivedi Yogesh Kumar, Rana Nripendra, Banita Lal y Michael Williams. 2015. "Consumer Adoption of Internet Banking in Jordan: Examining the Role of Hedonic Motivation, Habit, Self-efficacy and Trust". Journal of Financial Services Marketing 20 (2): 145-157. 10.1057/fsm.2015.5.

Al-Jabri, Ibrahim, y Sohail Sadiq. 2012. "Mobile Banking Adoption: Application of Diffusion of Innovation Theory". Journal of Electronic Commerce Research 13 (4): 379-391.

Al-Smadi, Mohammad. 2012. "Factors Affecting Adoption of Electronic Banking: An Analysis of the Perspectives of Banks' Customers". International Journal of Business and Social Science 3 (17): 294-300. https://bit.ly/3bW93g9.

Boateng, Henry, Adam Diyawu, Abednego Okoe y Thomas Anning-Dorson. 2016. “Assessing the Determinants of Internet Banking Adoption Intentions: A Social Cognitive Theory Perspective". Computers in Human Behavior 65: 468-478. 10.1016/j.chb.2016.09.017.

Bojan, Daniela, Simona Mutu y Dragos Păun. 2010. "Electronic Banking-Advantages for Financial Services Delivery". Annals of Faculty of Economics 1 (2): 672-677.

Chan, Siu Cheng. 2004. "Understanding Internet Banking Adoption and Use Behavior: A Hong Kong Perspective". Journal of Global Information Management (JGIM) 12 (3): 2143. 10.4018/jgim.2004070102. 
Chauhan, Vikas, Rambalak Yadav y Vipin Choudhary. 2019. "Analyzing the Impact of Consumer Innovativeness and Perceived Risk in Internet Banking Adoption". International Journal of Bank Marketing 37 (1) 323-339. 10.1108/IJBM-02-2018-0028.

Diniz, Eduardo, Roseli Morena Porto y Tomi Adachi. 2005. "Internet Banking in Brazil: Evaluation of Functionality, Reliability and Usability". The Electronic Journal of Information Systems Evaluation 8 (1): 41-50.

Garson, David. 1991. "A Comparison of Neural Network and Expert Systems Algorithms with Common Multivariate Procedures for Analysis of Social Science Data”. Social Science Computer Review 9 (3): 399-434. 10.1177/089443939100900304.

Gerrard, Philip, Barton Cunningham y James Devlin. 2006. "Why Consumers Are Not Using Internet Banking: A Qualitative Study”. Journal of Services Marketing 20 (3): 160-168. 10.1108/08876040610665616.

Hanafizadeh, Payam, Byron Keating y Hamid Khedmatgozar. 2014. “A Systematic Review of Internet Banking Adoption". Telematics and Informatics 31 (3): 492-510. 10.1016/j. tele.2013.04.003.

Heidenreich, Sven, y Patrick Spieth. 2013. "Why Innovations Fail-The Case Of Passive and Active Innovation Resistance". International Journal of Innovation Management 17 (05): 135-145. 10.1142/S1363919613500217.

Hoehle, Hartmut, Eusebio Scornavacca y Sid Huff. 2012. "Three Decades of Research on Consumer Adoption and Utilization of Electronic Banking Channels: A Literature Analysis". Decision Support Systems 54 (1): 122-132. 10.1016/j.dss.2012.04.010.

Hornik, Kurt. 1993. "Some New Results on Neural Network Approximation”. Neural Networks 6 (8): 1069-1072. 10.1016/S0893-6080(09)80018-X.

Joseph, Mathew, Cindy McClure y Beatriz Joseph. 1999. "Service Quality in the Banking Sector: The Impact of Technology on Service Delivery". International Journal of Bank Marketing 17 (4): 182-191. https://bit.ly/2FkSm28.

King, Brett. 2012. Bank 3.0: Why Banking is no Longer Somewhere you go but Something you Do. Nueva Jersey: John Wiley \& Sons.

Kleijnen, Mirella, Nick Lee y Martin Wetzels. 2009. "An Exploration of Consumer Resistance to Innovation and its Antecedents". Journal of Economic Psychology 30 (3): 344-357. 10.1016/j.joep.2009.02.004.

Laukkanen, Tommi. 2016. "Consumer Adoption vs Rejection Decisions in Seemingly Similar Service Innovations: The Case of the Internet and Mobile Banking”. Journal of Business Research 69 (7): 2432-2439. 10.1016/j.jbusres.2016.01.013.

Lee, Ming Chi. 2009. "Factors Influencing the Adoption of Internet Banking: An Integration of TAM and TPB with Perceived Risk and Perceived Benefit". Electronic Commerce Research and Applications 8 (3): 130-141. 10.1016/j.elerap.2008.11.006.

Martins, Carolina, Tiago Oliveira y Ales Popovič. 2014. "Understanding the Internet Banking Adoption: A Unified Theory of Acceptance and Use of Technology and Perceived Risk 
Application”. International Journal of Information Management 34 (1): 1-13. 10.1016/j. ijinfomgt.2013.06.002.

Nami, Mohammad Reza. 2009. "E-banking: Issues and Challenges". En 10th ACIS International Conference on Software Engineering, Artificial Intelligences, Networking and Parallel/Distributed Computing, 263-266. Institute of Electrical and Electronics Engineers, Daegu. 10.1109/SNPD.2009.60.

Pikkarainen, Tero, Kari Pikkarainen, Heikki Karjaluoto y Seppo Pahnila. 2004. "Consumer Acceptance of Online Banking: An Extension of the Technology Acceptance Model". Internet Research 4 (3): 224-235. 10.1108/10662240410542652.

Rotchanakitumnuai, Siriluck, y Mark Speece. 2003. "Barriers to Internet Banking Adoption: A Qualitative Study among Corporate Customers in Thailand". International Journal of Bank Marketing 21 (6/7): 312-323. 10.1108/02652320310498465.

Shaikh, Aijaz, y Heikki Karjaluoto. 2015. "Mobile Banking Adoption: A Literature Review". Telematics and Informatics 32 (1): 129-142. 10.1016/j.tele.2014.05.003.

Sharma, Sonia. 2016. "A Detail Comparative Study on E-Banking vs Traditional Banking". International Journal of Advanced Research 2 (7): 302-307. https://bit.ly/2Fn4SOC.

Tan, Margaret, y Teo Thompson. 2000. "Factors Influencing the Adoption of Internet Banking”. Journal of the Association for information Systems 1 (1): 5. 10.17705/1jais.00005.

Vives, Xavier. 2019. “Digital Disruption in Banking”. Annual Review of Financial Economics 11: 243-272. https://doi.org/10.1146/annurev-financial-100719-120854.

Welsch, Heinz, y Susana Ferreira. 2014. "Environment, Well-Being, and Experienced Preference”. Working Paper 367 (14). Universitat Oldenburg, Oldenburg. http://hdl.handle. net/10419/105055.

Wu, Jen-Her, Tzyh-Li Hsia y Michael Heng. 2006. "Core Capabilities for Exploiting Electronic Banking". Journal of Electronic Commerce Research 7 (2): 111-119. https://bit. ly/33qPQ25.

Yu, Chian-son. 2012. "Factors Affecting Individuals to Adopt Mobile Banking: Empirical Evidence from the UTAUT Model". Journal of Electronic Commerce Research 13 (2): 104-121. https://bit.ly/3mfhgR8.

Zhao, Ding, Ruolei Gu, Ping Tang, Qiwei Yang y Yue-Jia Luo. 2016. "Incidental Emotions Influence Risk Preference and Outcome Evaluation". Psychophysiology 53 (10): 15421551. 10.1111/psyp.12694.

Zhang, Yun, Qingxiong Weng y Nan Zhu. 2018. "The Relationships Between Electronic Banking Adoption And its Antecedents: A Meta-Analytic Study of the Role of National Culture". International Journal of Information Management 40: 76-87. 10.1016/j.ijinfomgt.2018.01.015.

Zolait, Ali Hussein, y Ainin Sulaiman. 2017. "The Influence of Communication Channels on Internet Banking Adoption". Asian Journal of Business Accounting. Hong Kong (1\&2): 115-134. https://bit.ly/2Rtb0ae. 